Pacific Journal of Mathematic 


\title{
COUNTING SUBGROUPS AND TOPOLOGICAL GROUP TOPOLOGIES
}

\author{
Shiferaw Berhanu, W. W. Comfort and J. D. Reid
}

Let $G$ be an Abelian group with $|G|=\alpha \geq \omega, \mathscr{S}(G)$ the set of subgroups of $G, \mathscr{B}$ the set of totally bounded topological group topologies on $G, \mathscr{M}(\gamma)$ the set of topological group topologies $\mathscr{T}$ for which the character $(=$ local weight) of $\langle G, \mathscr{T}\rangle$ is equal to $\gamma \geq \omega$, and $\mathscr{B}(\gamma)=$ $\mathscr{B} \cap \mathscr{M}(\gamma)$. We prove algebraic results and topological results, as follows.

Algebra. Either $|\mathscr{S}(G)|=2^{\alpha}$ or $|\mathscr{S}(G)|=\alpha$. If $|\mathscr{S}(G)|=\alpha$ then $\alpha=\omega$. We describe and characterize those (countable) $G$ such that $|\mathscr{S}(G)|=\omega$, and we give several examples.

Topology. If $\gamma<\log (\alpha)$ or $\gamma>2^{\alpha}$, then $\mathscr{B}(\gamma)=\varnothing$; otherwise $|\mathscr{B}(\gamma)|=2^{\alpha \cdot \gamma}$. If $\gamma>2^{\alpha}$ then $\mathscr{M}(\gamma)=\varnothing$; if $\log (\alpha)<\gamma \leq 2^{\alpha}$ then $|\mathscr{M}(\gamma)|=2^{\alpha}{ }^{\gamma}$; and if $\omega \leq \gamma \leq \alpha$ then $|\mathscr{M}(\gamma)|=2^{\alpha}$.

0. Introduction and motivation. As a reading of the Synopsis may suggest, this work originated with the authors' interest in the following questions: Given an infinite Abelian group, how many topological group topologies does $G$ possess? Of these, how many may be chosen pairwise non-homeomorphic? How many metrizable? How many totally bounded? How many totally bounded and metrizable? We approached the latter questions through a result from [6] which gives a one-to-one order-preserving correspondence between the set $\mathscr{B}(G)$ of totally bounded topological group topologies for $G$ and the set of point-separating subgroups of the homomorphism group $\operatorname{Hom}(G, \mathbf{T})$. Thus it became natural-indeed necessary-to count the number of subgroups of a group of the form $\operatorname{Hom}(G, \mathbf{T})$. In $\S \S 1$ and 2 , which we believe have algebraic interest quite independent of their topological roots, we do a bit more: We show that every uncountable Abelian group $G$ has $2^{|G|}$ subgroups, and we describe in some detail the fine algebraic structure of what we call $\omega$-groups. These are by definition the (necessarily countable) Abelian groups $G$ with fewer then $2^{|G|}$ subgroups; we show that each $\omega$-group has exactly $\omega$-many subgroups, and we describe the relationship between the $\omega$-groups and the so-called q.d. groups of Beaumont and Pierce [2].

The algebraic analysis of $\S 1$, together with the result cited from [6], allows us to describe some gross features of the partially ordered sets $\mathscr{B}(G)$. Here our work is sufficiently coarse that the various cardinal 
numbers we associate with the sets $\mathscr{B}(G)$ are determined by $|G|$ alone-that is, they are unaffected by algebraic properties of the (Abelian) groups $G$.

Sections 1,2 and 3 are algebraic in nature. Of these, only $\S 1$ is required for the topological analysis of $\S \S 4,5$ and 6.

Some other authors have considered the question of the number of topological group topologies on an Abelian group. For example Kiltinen [19], [20], using methods from the theory of topological fields, showed that the obvious upper bound $2^{2^{|G|}}$ is achieved; this result was corroborated by Podewski [23], [24]. The papers [6], [7] and [8] (7.2) contain partial results concerning totally bounded topologies; and Fuchs [11], [12] (47.6) has noted that for every $\alpha \geq \omega$ there is an Abelian group $G$ with $|G|=2^{\alpha}$ and with $2^{\alpha}$ pairwise non-homeomorphic compact topological group topologies. While the present work, some of whose results we announced in [3], was being completed, we learned from Dieter Remus that in his forthcoming doctoral dissertation [26] he will present, using methods quite different from ours, results equivalent to our Corollary 5.7(a) and the case $\gamma=\omega$ of Theorem 5.3(a), as well as other lattice-theoretic theorems unrelated to those of this paper.

1. Concerning $\mathscr{S}(G)$ when $|G|>\omega$. Throughout this work we denote by $\mathscr{S}(G)$ the set of subgroups of the Abelian group $G$.

The symbols $\mathbf{Z}, \mathbf{Q}$ and $\mathbf{R}$ denote, respectively, the integers, the rationals and the reals, and $\mathbf{T}$ and $\mathbf{W}$ denote, respectively, the circle and its torsion subgroup.

Most of the groups we consider are Abelian. The identity or neutral element is usually denoted 0 . Occasionally when specificity is called for, the identity of a group $G$ (or $G_{i}$, etc.) will be denoted by $e_{G}$ (or $e_{i}$, etc.). Of course the identity of $\mathbf{T}$ is $e_{\mathrm{T}}=1$.

The direct sum of a set $\left\{G_{i}: i \in I\right\}$ of groups is denoted $\oplus_{i \in I} G_{i}$; if all $G_{i}$ are isomorphic to a fixed group $G$, we sometimes write $\oplus_{I} G$ in place of $\oplus_{i \in I} G_{i}$. Occasionally we identify $\bigoplus_{i \in I} G_{i}$ with the group

$$
\left\{x \in \prod_{i \in I} G_{i}:\left|\left\{i \in I: x_{i} \neq e_{\imath}\right\}\right|<\omega\right\} .
$$

1.1. TheOREM. Let $G$ be an Abelian group with $|G|=\alpha>\omega$. Then $G$ contains a subgroup of the form $\oplus_{\xi<\alpha} G_{\xi}$ with each $G_{\xi}$ cyclic, $\left|G_{\xi}\right|>1$.

Proof. We recall first, perhaps from Fuchs [12] (28.2), a theorem of Kulikov: If $K$ is a pure subgroup of $G$ such that $G / K$ is the direct sum $\bigoplus_{i \in I} C_{i}$ with each $C_{i}$ cyclic, then $K$ is a direct summand of $G$ (and hence we have the isomrphism $G \cong K \times \bigoplus_{i \in I} C_{i}$ ). 
We denote by $t(G)$ the torsion subgroup of $G$, and we consider two cases.

Case $1 .|G / t(G)|=\alpha$. Let $[A]$ be the subgroup of $G / t(G)$ generated by a maximal independent subset $A$ of $G / t(G)$. From $|G / t(G)|=\alpha>\omega$ we have $|[A]|=|A|=\alpha$ and hence $[A] \approx \oplus_{\alpha} \mathbf{Z}$. There is $H \in \mathscr{S}(G)$ such that $[A]=H / t(G)$, and since $t(G)$ is pure in $H$ we have

$$
G \supset H \cong t(G) \times \bigoplus_{\alpha} Z
$$

from Kulikov's theorem, as required.

Case 2. $|G / t(G)|<\alpha$. In this case $|t(G)|=\alpha$ and we assume without loss of generality that $G=t(G)$, i.e., $G$ is a torsion group. For each prime $p$ let $G_{p}$ denote the $p$-primary subgroup of $G$, let $B_{p}$ be a basic subgroup of $G_{p}$, and set $B=\oplus_{p} B_{p}$. If $|B|=\alpha$, then since $\alpha>\omega$ and $B$ has the form $B=\bigoplus_{i \in I} C_{i}$ with each $C_{i}$ cyclic we have $|I|=\alpha$ and the proof is complete. If $|B|<\alpha$ then the (divisible) quotient $D=G / B$ satisfies $|D|=\alpha$ and again from $\alpha>\omega$ we have, writing $D[p]=\{x+B \in D: p x \in B\}$ for $p$ prime and $E=\oplus_{p} D[p]$, that $|E|=\alpha$. Since $E$ has the form $E=\oplus_{i \in I} C_{i}$ with each $C_{i}^{p}$ cyclic, from $\alpha>\omega$ follows $|I|=\alpha$. There is $H \in \mathscr{S}(G)$ such that $E=H / B$, and since $B$ is pure in $H$ we have again

$$
G \supset H \cong B \times \bigoplus_{i \in I} C_{i}
$$

from Kulikov's theorem, as required.

The following is an immediate consequence of 1.1 .

1.2. Corollary. Let $G$ be an Abelian group with $|G|=\alpha>\omega$. Then $|\mathscr{S}(G)|=2^{\alpha}$.

Theorem 1.1 allows us to analyze in some detail the structure of the partially ordered set $\mathscr{S}(G)$. The following notation is useful.

Definition. Let $G$ be an Abelian group and let $\gamma \geq \omega$. Then $\mathscr{S}(G, \gamma)$ $=\{A \in \mathscr{S}(G):|A|=\gamma\}$.

In the following result, crucial to our investigations in topology below, the symbols $\mathscr{A}$ and $\mathscr{W}$ are chosen as mnemonics for "anti-chain" and "well-ordered", respectively.

1.3. Corollary. Let $G$ be an Abelian group with $|G|=\alpha>\omega$, and let $\omega \leq \gamma \leq \alpha$. Define $\bar{\gamma}=\min \left\{\gamma^{+}, \alpha\right\}$. Then 
(a) there is $\mathscr{A} \subset \mathscr{S}(G, \gamma)$ with $|\mathscr{A}|=\alpha^{\gamma}$ such that no two elements of $\mathscr{A}$ are comparable (with respect to set-theoretic inclusion); and

(b) there are $\mathscr{W}, \mathscr{W}^{*} \subset \mathscr{S}(G, \gamma)$ with $|W|=\bar{\gamma}$ and $\left|W^{*}\right|=\gamma$ such that $\mathscr{W}$ is well-ordered and $\mathscr{W}^{*}$ is anti-well-ordered (with respect to set-theoretic inclusion). $\xi<\alpha$.

Proof. Using 1.1 we write $G \supset \oplus_{\xi<\alpha} G_{\xi}$ with $2 \leq\left|G_{\xi}\right| \leq \omega$ for all

(a) We note that for (every set of cardinality) $\alpha$ there is $\mathscr{F} \subset \mathscr{P}(\alpha)$ such that $|\mathscr{F}|=\alpha^{\gamma}$, each $F \in \mathscr{F}$ satisfies $|F|=\gamma$, and $\mathscr{F}$ is an antichain with respect to set-theoretic inclusion. (Since $|\gamma \times \alpha|=\alpha$, it is enough to find such a family of subsets of $\gamma \times \alpha$; the family $\left\{\operatorname{Graph}(f): f \in \alpha^{\gamma}\right\}$ is as required.) Let $\mathscr{F}$ be such a family of subsets of $\alpha$ and for $F \in \mathscr{F}$ set

$$
A(F)=\bigoplus_{\xi \in F} G_{\xi} .
$$

Since $\left|G_{\xi}\right|>1$ for all $\xi<\alpha$, it is clear that the family $\mathscr{A}=\{A(F)$ : $F \in \mathscr{F}\}$ is as required.

(b) We write $\alpha=\bigcup_{\eta<\alpha} A_{\eta}$ with $\left\{A_{\eta}: \eta<\alpha\right\}$ a family of pairwise disjoint sets and with each $\left|A_{\eta}\right|=\gamma$, and we set $H_{\eta}=\oplus_{\xi \in A_{\eta}} G_{\xi}$ for $\eta<\alpha$. Then each $\left|H_{\eta}\right|=\gamma$, and $G \supset \bigoplus_{\eta<\alpha} H_{\eta}$. Now we set

and

$$
W_{\zeta}=\bigoplus_{\eta \leq \zeta} H_{\eta} \quad \text { for } \zeta<\bar{\gamma},
$$

$$
W_{\zeta}^{*}=\bigoplus_{\zeta \leq \eta<\gamma} H_{\eta} \quad \text { for } \zeta<\gamma \text {. }
$$

For $\zeta<\bar{\gamma}$ we have $\gamma=\left|H_{0}\right| \leq\left|W_{\xi}\right| \leq \gamma \cdot|\zeta|=\gamma$, and for $\zeta<\gamma$ we have

$$
\gamma=\left|\bigoplus_{\eta<\gamma} H_{\eta}\right| \geq\left|W_{\zeta}^{*}\right|=\gamma \cdot|\gamma \backslash \zeta|=\gamma \cdot \gamma=\gamma
$$

Thus the families $\mathscr{W}=\left\{W_{\zeta}: \zeta<\bar{\gamma}\right\}$ and $\mathscr{W}^{*}=\left\{W_{\zeta}^{*}: \zeta<\gamma\right\}$ are as required.

The following result (which we need below) was apparently first stated, and proved elegantly, by Kakutani [18]; see also Fuchs [12] (47.6). Most of the proofs of this theorem known to the authors require separate consideration of the two cases $\alpha=\omega, \alpha>\omega$. As Lewis C. Robertson has remarked in conversation, the case $\alpha>\omega$ is immediate from 1.1 above.

1.4. TheOREM. Let $G$ be an Abelian group with $|G|=\alpha \geq \omega$. Then $|\operatorname{Hom}(G, \mathbf{T})|=2^{\alpha}$. 
2. $\omega$-groups. We have seen that groups $G$ of uncountable cardinality $\alpha$ have many subgroups - indeed contain within themselves direct sums of $\alpha$-many subgroups - and that if $G$ has fewer than $2^{\alpha}$ subgroups, then $|G|=\alpha=\omega$. We now define an $\omega$-group to be an Abelian group, necessarily countable, that has fewer than $2^{\omega}$ subgroups. It is clear that subgroups and homomorphic images of $\omega$-groups are $\omega$-groups, and that such groups cannot contain direct sums of infinitely many non-trivial subgroups. We will use these facts with little explicit reference in our discussion.

2.1. Lemma. Let $G=A \oplus B$ be the direct sum of its subgroups $A$ and $B$. Then the complements of $A$ in $G$ are in one-to-one correspondence with the elements of $\operatorname{Hom}(B, A)$.

This result is well-known. We merely remark that if $f: B \rightarrow A$ is a homomorphism then the corresponding complement, $B(f)$, of $A$ in $G$ is the "graph" of $f$ :

$$
B(f)=\{f(b)+b: b \in B\}
$$

2.2. COROLlaRY. Let $H$ be a subgroup of the group $G$ and let $\mathscr{D}(H)=$ $\{K \in \mathscr{S}(G): H \cap K=0\}$. Then

$$
|\mathscr{D}(H)| \leq \sum\{|\operatorname{Hom}(\bar{K}, H)|: \bar{K} \in \mathscr{S}(G / H)\} .
$$

Proof. Define an equivalence relation in $\mathscr{D}(H)$ by

$$
K \sim K^{\prime} \quad \text { if and only } H+K=H+K^{\prime} .
$$

Clearly the number of equivalent classes does not exceed $|\mathscr{S}(G / H)|$. Fix $K_{0} \in \mathscr{D}(H)$. Then $K_{0} \cong\left(H+K_{0}\right) / H=\bar{K} \in \mathscr{S}(G / H)$ and $K^{\prime} \sim K_{0}$ means that $K^{\prime}$ is a complement of $H$ in $H+K_{0}$. Thus the equivalence class of $K_{0}$ has cardinality $\left|\operatorname{Hom}\left(K_{0}, H\right)\right|=|\operatorname{Hom}(\bar{K}, H)|$, and the result follows.

It is clear now that the torsion subgroup of an $\omega$-group has only finitely many primary components, each of which has a finite basic subgroup, and the torsion-free quotient has finite rank. On the other hand the torsion-free group, $\mathbf{Q}$, of rational numbers has, as is well known, $2^{\omega}$ subgroups; and since $\operatorname{Hom}\left(\mathbf{Z}\left(p^{\infty}\right), \mathbf{Z}\left(p^{\infty}\right)\right)$ is isomorphic to the $p$-adic integers, the lemma above implies that $\mathbf{Z}\left(p^{\infty}\right) \oplus \mathbf{Z}\left(p^{\infty}\right)$ has $2^{\omega}$ subgroups as well. Thus the situation is perhaps not completely trivial. We start with 
the following:

2.3. Proposition. A torsion $\omega$-group $G$ has the form

$$
G=H \oplus \bigoplus_{p \in F} \mathbf{Z}\left(p^{\infty}\right)
$$

where $H$ is a finite group and $F$ is a finite set of primes.

Proof. We have seen that $G$ has but finitely many primary components $G_{p}$, each having a finite basic subgroup $B_{p}$. Thus $G_{p}=B_{p} \oplus D_{p}$ with $D_{p}$ divisible. By $2.1, D_{p}$ is either 0 or $\mathbf{Z}\left(p^{\infty}\right)$ so $G=\bigoplus_{p \in F^{\prime}}\left(B_{p} \oplus D_{p}\right)$, with $F^{\prime}$ a finite set of primes. Thus $G$ has the form described, with $H$ the direct sum of the $B_{p}$ and $F$ the set of primes for which $D_{p} \neq 0$.

2.4. LEMMA. Let $H$ be a finite subgroup of an infinite Abelian group $G$. If $G / H$ is an w-group then $|\mathscr{S}(G)| \leq|\mathscr{S}(G / H)|$. In particular, $G$ is an $\omega$-group.

Proof. We use induction on the order, $h$, of $H$. There being nothing to prove if $h=1$, assume first that $h$ is not prime. Then $H$ contains a proper non-trivial subgroup $L$ and $H / L$ is a finite subgroup of the infinite Abelian group $G / L$ with quotient isomorphic to $G / H$, hence an $\omega$-group. By the induction hypothesis we conclude that

$$
|\mathscr{S}(G / L)| \leq|\mathscr{S}(G / L / H / L)|=|\mathscr{S}(G / H)|<2^{\omega} .
$$

Thus $G / L$ is an $\omega$-group. Applying the induction hypothesis once more we obtain

$$
|\mathscr{S}(G)| \leq|\mathscr{S}(G / L)| \leq|\mathscr{S}(G / H)|
$$

as required.

Now suppose that $h$ is prime. Then every subgroup of $G$ either contains $H$ or is disjoint from it, and the set of those that contain $H$ is in one-to-one correspondence with the set of subgroups of $G / H$, so there are $|\mathscr{S}(G / H)|$ of these. If $W$ is a subgroup of $G$ disjoint from $H$, then since $h H=0$ we have $\operatorname{Hom}(W, H) \cong \operatorname{Hom}(W / h W, H)$. Now $W / h W$ is bounded, hence a direct sum of cyclic groups, and is an $\omega$-group since it is an image of $W$ and $W$ is isomorphic to a subgroup of the $\omega$-group $G / H$. Hence there can be but finitely many cyclic summands of $W / h W$ so it, and with it $\operatorname{Hom}(W, H)$, is finite.

It now follows from Corollary 2.2 that the number of subgroups of $G$ disjoint from $H$ does not exceed $|S(G / H)|$. Altogether then we clearly have $|\mathscr{S}(G)| \leq|\mathscr{S}(G / H)|$, as required. 
2.5. THEOREM. A torsion group $G$ is an $\omega$-group if and only if

$$
G=H \oplus \bigoplus_{p \in F} \mathbf{Z}\left(p^{\infty}\right)
$$

with $\mathrm{H}$ a finite group and $\mathrm{F}$ a finite set of primes.

Proof. By Proposition 2.3, a torsion $\omega$-group has the indicated form. To establish the converse it suffices by Lemma 2.4 to show that $\bigoplus_{p \in F} \mathbf{Z}\left(p^{\infty}\right), F$ finite, has but $\omega$-many subgroups. This is clear since a subgroup of such a group has but countably many choices for each of its (necessarily finitely many) primary components.

\subsection{COROLlaRY. If $G$ is a torsion $\omega$-group then $|\mathscr{S}(G)| \leq \omega$.}

Proof. This follows immediately from 2.4 and 2.5.

Now suppose that $G$ is a torsion-free $\omega$-group. We have already observed that $G$ must have finite rank. Let $X_{0}$ be the subgroup of $G$ generated by a maximal independent set. Then $G / X_{0}$ is a torsion $\omega$-group so has the form $H \oplus D$ with $H$ finite and $D$ divisible, as in Theorem 2.5. Let $X$ be the subgroup of $G$ satisfying $X / X_{0}=H$. Then $G / X=D$ and since $H$ is finite and $X_{0}$ is free, $X$ is free. These remarks establish half of the following statement.

2.7. TheOREM. A torsion-free group $G$ is an $\omega$-group if and only if there is an exact sequence

$$
0 \rightarrow X \rightarrow G \rightarrow \prod_{p \in F} \mathbf{Z}\left(p^{\infty}\right) \rightarrow 0
$$

with $X$ free of finite rank and $F$ a finite set of primes.

Proof. To complete the proof it suffices to show that if a sequence of the form (*) exists for $G$ then in fact $G$ has only $\omega$-many subgroups. Let $M$ be any subgroup of $G$, and let $X(M)=X \cap M$; then $M / X(M)$ is a torsion subgroup of $G / X(M)$. Moreover, $M$ is uniquely determined by the pair $(X(M), M / X(M))$. It is clear that there are only countably many subgroups of $X$. Thus it suffices to show that, for each subgroup $X_{1}$ of $X$, the torsion subgroup $t\left(G / X_{1}\right)$ has at most countably many subgroups. Put

$$
X_{1}^{*}=\{g \in G: n g \in X \text {, for some } n \neq 0, n \in \mathbf{Z}\},
$$


so $X_{1}^{*}$ is the pure subgroup of $G$ generated by $X_{1}$. Then $t\left(G / X_{1}\right)=X_{1}^{*} / X_{1}$. Note that $\left(X_{1}^{*} \cap X\right) / X_{1}$ is finitely generated and torsion, hence is finite. Moreover

$$
X_{1}^{*} /\left(X_{1}^{*} \cap X\right) \cong\left(X_{1}^{*}+X\right) / X \subset G / X \cong \prod_{p \in F} \mathbf{Z}\left(p^{\infty}\right)
$$

so $X_{1}^{*} /\left(X_{1}^{*} \cap X\right)$ is an $\omega$-group. Now by Lemma 2.4 we have

$$
\left|\mathscr{S}\left(X_{1}^{*} / X_{1}\right)\right| \leq\left|\mathscr{S}\left(X_{1}^{*} /\left(X_{1}^{*} \cap X\right)\right)\right| \leq \omega,
$$

as required.

\subsection{COROLlary. Let $G$ be a torsion-free $\omega$-group. Then}

$$
|\mathscr{S}(G)|=\omega .
$$

Groups which have the general structure exhibited in Theorem 2.7-i.e. modulo some free subgroup the group is divisible-were first studied by Beaumont and Pierce [2] and were called q.d. groups (quotient divisible groups). It is perhaps of interest that any torsion-free group of infinite rank is a q.d. group ([25]), but for groups of finite rank this is a restriction. If $G$ is torsion-free of finite rank $n$ and $X_{0}$ is a free subgroup of $G$ such that $G / X_{0}$ is divisible, then for any free subgroup $X$ of $G$ of rank $n, G / X$ is the direct sum of a finite group and a divisible group, and the structure of the divisible group is independent of the choice of $X$. These results are not difficult to prove; we refer the reader to [2], [22] and [25] for proofs and further discussion of q.d. groups. Our point here is that the set $F$ of primes arising for the torsion-free $\omega$-group $G$ in Theorem 2.7 is an invariant of $G$; it does not depend on the choice of $X$. We write $F(G)$ for this set of primes.

Finally we consider the structure of an arbitrary $\omega$-group $G$. Its torsion subgroup, $t(G)$, is an $\omega$-group, so is described by Theorem 2.5 . It is well known [12] that in such a case $t(G)$ is a direct summand, so that

$$
G=t(G) \oplus G_{0}
$$

with $G_{0}$ a torsion-free $\omega$-group, hence described by Theorem 2.7. These observations yield one half of:

2.9. TheOREM. An Abelian group $G$ is an $\omega$-group if and only if $G=t(G) \oplus G_{0}$, where

(i) $t(G)=H \oplus \prod_{p \in F} \mathbf{Z}\left(p^{\infty}\right)$ with $H$ a finite group and $F$ a finite set of primes;

(ii) $G_{0}$ is a torsion-free $\omega$-group; and

(iii) $F\left(G_{0}\right) \cap F=\varnothing$. 
Proof. The fact that an $\omega$-group has the indicated form is immediate from the remarks above together with the observation that, were $p \in$ $F\left(G_{0}\right) \cap F$ then $\mathbf{Z}\left(p^{\infty}\right) \oplus \mathbf{Z}\left(p^{\infty}\right)$ would appear in $G / X$ where $X$ is a free subgroup in $G_{0}$ as described in Theorem 2.7. But no image of an $\omega$-group can contain such a subgroup so $F\left(G_{0}\right)$ and $F$ are indeed disjoint.

For the converse we show that any group $G$ with the indicated structure has at most countably many subgroups, so in particular is an $\omega$-group. Let $G^{\prime}$ be a subgroup of $G$. Then the torsion subgroup $t\left(G^{\prime}\right)$ of $G^{\prime}$ is one of the countably many subgroups of $t(G)$. Put $t\left(G^{\prime}\right)=V$. We will count the number of subgroups of $G$ with maximal torsion subgroup equal to $V$. This is the same as counting the number of torsion-free subgroups of $G / V$. Now $t(G) / V$ is the torsion subgroup of $G / V$ and $t(G) / V$ has the same form as $t(G)$ itself-i.e. a finite group plus a direct sum of finitely many $\mathbf{Z}\left(p^{\infty}\right)$ 's, none repeated. The probem is clearly equivalent then to counting the number of torsion-free subgroups of a group $G$ of the form $G=t(G) \oplus G_{0}$ as above. Finally, since $|\mathscr{S}(G / t(G))|$ $=\left|\mathscr{S}\left(G_{0}\right)\right|$ is countable it suffices by 2.2 to show that, for each subgroup $K$ of $G_{0}, \operatorname{Hom}(K, t(G))$ is countable.

From the form of $t(G)$ we have

$$
\operatorname{Hom}(K, t(G)) \cong \operatorname{Hom}(K, H) \oplus \prod_{p \in F} \operatorname{Hom}\left(K, \mathbf{Z}\left(p^{\infty}\right)\right)
$$

and since $H$ is finite, of order $h$ say, $\operatorname{Hom}(K, H) \cong \operatorname{Hom}(K / h K, H)$. Since $K$ is torsion-free of finite rank, $K / h K$ is finite, so $\operatorname{Hom}(K, H)$ is finite. From 2.7 we have an exact sequence

$$
0 \rightarrow X \rightarrow G_{0} \rightarrow \prod_{p \in F\left(G_{0}\right)} \mathbf{Z}\left(p^{\infty}\right) \rightarrow 0
$$

and if $X_{1}=K \cap X$ then $K / X_{1} \cong(K+X) / X \subset G_{0} / X$ so $K / X_{1} \cong$ $\Pi_{p \in F\left(G_{0}\right)} \mathbf{Z}\left(p^{k_{p}}\right)$ with $0 \leq k_{p} \leq \infty$. Now if $p_{1} \in F$, the exact sequence

$$
0 \rightarrow X_{1} \rightarrow K \rightarrow \prod \mathbf{Z}\left(p^{k_{p}}\right) \rightarrow 0
$$

yields

$$
\begin{aligned}
0 & \rightarrow \operatorname{Hom}\left(\prod_{p \in F\left(G_{0}\right)} \mathbf{Z}\left(p^{k_{p}}\right), \mathbf{Z}\left(p_{1}^{\infty}\right)\right) \\
& \rightarrow \operatorname{Hom}\left(K, \mathbf{Z}\left(p_{1}^{\infty}\right)\right) \rightarrow \operatorname{Hom}\left(X_{1}, \mathbf{Z}\left(p_{1}^{\infty}\right)\right) .
\end{aligned}
$$

Since $p_{1} \notin F\left(G_{0}\right), \operatorname{Hom}\left(\Pi \mathbf{Z}\left(p^{k_{p}}\right), \mathbf{Z}\left(p_{1}^{\infty}\right)\right)=0$ so we have the inclusion

$$
0 \rightarrow \operatorname{Hom}\left(K, \mathbf{Z}\left(p_{1}^{\infty}\right)\right) \rightarrow \operatorname{Hom}\left(X_{1}, \mathbf{Z}\left(p_{1}^{\infty}\right)\right) ;
$$


and since $X_{1}$ is free of finite rank, $\operatorname{Hom}\left(X_{1}, \mathbf{Z}\left(p^{\infty}\right)\right)$ is countable. Thus $\operatorname{Hom}\left(K, \mathbf{Z}\left(p_{1}^{\infty}\right)\right)$ is countable (cf. also [12] (47.1)). It follows that $\operatorname{Hom}(K, t(G))$ is countable. This proves the theorem.

We may consolidate the previous three theorems in the following.

2.10. THEOREM. An Abelian group $G$ is an w-group if and only if there is an exact sequence

$$
0 \rightarrow W \rightarrow G \rightarrow \prod_{p \in S} \mathbf{Z}\left(p^{\infty}\right) \rightarrow 0
$$

with $W$ a finitely generated group and $S$ a finite set of primes.

From our discussion, we also have

\subsection{Corollary. If $G$ is an infinite $\omega$-group then $|\mathscr{S}(G)|=\omega$.}

3. Torsion-free $\omega$-groups. ExAMPLES. There is an interesting example (see Arnold [1] (Example 2.7)) of a torsion-free group of finite rank $n$ which is strongly indecomposable, homogeneous with type the type of $\mathbf{Z}$ and in which every subgroup of rank less than $n$ is free. Indeed such a group is constructed for each $n$ and Pierce [1] (Exercise 2.5) has shown that, even when $n=2$, there are $2^{\omega}$ such groups, no two isomorphic with each other. It follows from Theorem 2.7 that these groups are $\omega$-groups, for, by construction, each has a full free subgroup with quotient isomorphic to $\mathbf{Z}\left(p^{\infty}\right), p$ a selected but essentially arbitrary prime. Thus the $\omega$-groups have perhaps surprisingly complicated structure.

On the other hand, Arnold's examples lie in the simplest class of $\omega$-groups, beyond the free groups, in the sense that the divisible quotient $G / X$ (cf. 2.7 ) is a single $\mathbf{Z}\left(p^{\infty}\right)$. We pause here to consider an arbitrary group of this kind, so that we have an exact sequence

$$
0 \rightarrow X \rightarrow G \rightarrow \mathbf{Z}\left(p^{\infty}\right) \rightarrow 0
$$

with $X$ free of rank $n$. Let $B$ be a pure subgroup of $G$ of rank $n-1$. Then $(B+X) / X \subset G / X \cong \mathbf{Z}\left(p^{\infty}\right)$, and it follows that either $B+X=G$ or $p^{k}(B+X) \subset X$ for some $k$. In the latter case, $B$ is free; in the former,

$$
G / B=(B+X) / X \cong X /(X \cap B)
$$

is finitely generated and torsion-free, hence free; thus we have $G=B \oplus C$ with $C \cong \mathbf{Z}$. Thus if $G$ is indecomposable then $B$ must be free. Note that 
in this case $G$ is homogeneous of type the type of $\mathbf{Z}$. These remarks follow Arnold's discussion quite closely.

In the general case, however, we put $W=\bigcap\{\operatorname{ker} f: f \in \operatorname{Hom}(G, \mathbf{Z})\}$, so $W$ is a pure (and fully invariant) subgroup of $G$. Since $G$ is of finite rank we can represent $W$ as an intersection of the kernels of a finite number of maps $f: G \rightarrow \mathbf{Z}$ and this leads to an imbedding of $G / W$ into a finite product of copies of $\mathbf{Z}$-i.e., into a free Abelian group. Hence $G / W$ is free so $G=W \oplus V$ with $V$ free and, evidently, $\operatorname{Hom}(W, \mathbf{Z})=0$. Now it is clear that $W$ is an $\omega$-group with the same property as $G$, namely $W / Y \cong \mathbf{Z}\left(p^{\infty}\right)$ for some free subgroup $Y$ of $W$. Since $W$ has no free summand we conclude by the remarks above that every subgroup of $W$ of smaller rank than that of $W$ is free and, $\operatorname{since} \operatorname{Hom}(W, \mathbf{Z})=0$, that $W$ is strongly indecomposable. Finally if $B$ is pure in $G$ and has rank $n-1$, say, then either $W \subset B$ (in which case $B=W \oplus C$ with $C$ free), or $W$ is not contained in $B$. In the latter case we have $B /(B \cap W) \cong(B+W) / W$ is free and $B \cap W$ is a proper pure subgroup of $W$, so $B \cap W$ is free. Hence $B$ is free. We summarize these remarks as follows.

3.1. Theorem. Suppose $0 \rightarrow X \rightarrow G \rightarrow \mathbf{Z}\left(p^{\infty}\right) \rightarrow 0$ is exact, with $X$ free of rank $n$. Put $W=\bigcap\{\operatorname{ker} f: f \in \operatorname{Hom}(G, \mathbf{Z})\}$. Then

(i) $G=W \oplus V, V$ free;

(ii) $W$ is strongly indecomposable, and every subgroup of $W$ of smaller rank is free; and

(iii) every subgroup of $G$ of rank less than $n$ that does not contain $W$ is free.

These examples suggest the following result, which gives an alternative description of the torsion-free $\omega$-groups. See [1] for background on types.

3.2. TheOREM. $A$ torsion-free Abelian group $G$ is an $\omega$-group if and only if there is an exact sequence

$$
0 \rightarrow B \rightarrow G \rightarrow A \rightarrow 0
$$

where $B$ is a free Abelian group of finite rank and $A$ is a rank $1 \omega$-group. The rank 1 w-groups are exactly those with idempotent type, zero at almost all primes.

Proof. The remark on rank $1 \omega$-groups is clear from Theorem 2.7. Moreover, if a sequence of the kind described exists for $G$, we may choose 
$C \subset A, C \cong Z$, such that $A / C=\prod_{p \in F} \mathbf{Z}\left(p^{\infty}\right)$ with $F$ a finite set of primes. Then with $X$ chosen so that $B \subset X \subset G$ and $X / B \cong C$ we have

$$
0 \rightarrow X \rightarrow G \rightarrow \prod_{p \in F} \mathbf{Z}\left(p^{\infty}\right) \rightarrow 0
$$

and $G$ is an $\omega$-group by 2.7 .

Conversely, if $G$ is a torsion-free $\omega$-group then it follows from Theorem 2.7 that the type set of $G$ is finite. In fact, if $\tau$ is the type with value $\infty$ at all $p \in F(G), 0$ otherwise, then it is clear that each non-zero element of $G$ has type less than or equal to $\tau$. Let $g \in G$ and write $t(g)$ for the type of $g$. Since $G$ is an $\omega$-group, $t(g)$ is zero at almost all primes, so if $t(g)$ is not the zero type then $t(g)$ is infinite at some prime. Clearly such primes lie in $F(G)$. Thus there are but finitely many possibilities for types of elements of $G$.

We assume now that $G$ has rank at least 2. Then if $x$ and $y$ are independent elements of $G$, the infimum of their types is the zero type: otherwise, as is clear, $\langle x\rangle_{*}+\langle y\rangle_{*}$ is not an $\omega$-group. Thus the inner type of $G$ is the zero type and since the type set is finite, the inner type is realized ([1] (1.7)). We conclude that $G$ has a pure free subgroup, other than 0 . Now choose $B$ to be a maximal proper pure free subgroup of $G$. By maximality of $B, G / B$ has rank 1 and is of course an $\omega$-group. This gives the desired exact sequence and completes the proof of the theorem.

We note that if $G$ is an $\omega$-group then the structure of the group $A$ in 3.1 is independent of the choice of the pure free subgroup $B$. Indeed the type of $A$ is the idempotent type which is $\infty$ at all $p \in F(G), 0$ otherwise.

As remarked earlier, Beaumont and Pierce have studied quotientdivisible groups in some detail [2] and in particular have constructed invariants, $\delta(G)$, for such groups $G$. These are sequences, indexed by the primes, of vector spaces $\delta(G)_{p}$ over the $p$-adic numbers. The $\omega$-groups are the q.d. groups of finite rank with $\delta_{p}(G)=0$ for almost all $p$, and one-dimensional when non-zero. Accordingly, Corollary 5.27 [2] gives a method of constructing strongly indecomposable $\omega$-groups in great variety and for any finite rank. We refer the interested reader to $\$ 5$ of [2] for details.

4. Topological notation, definitions, and results from the literature. Here we describe our set-theoretic and topological terminology and conventions, and we quote from the literature several results needed in $\S 5$ and $\S 6$.

For $X$ a set we write $\mathscr{P}(X)=\{A: A \subset X\}$.

For a cardinal number $\alpha \geq \omega$ we set $\log (\alpha)=\min \left\{\beta: 2^{\beta} \geq \alpha\right\}$. 
For $G$ an Abelian group and $\mathscr{T}$ a topology for $G$, the statement that $G=\langle G, \mathscr{T}\rangle$ is a topological group means that $\langle G, \mathscr{T}\rangle$ satisfies the Hausdorff separation property and the function $\langle a, b\rangle \rightarrow a b^{-1}$ from $G \times G$ to $G$ is continuous. As is well known (see for example [13] (8.4)), the requirement that $\mathscr{T}$ is a Hausdorff topology guarantees that $\langle G, \mathscr{T}\rangle$ is a completely regular space, i.e., a Tychonoff space.

A topological group $G$ is said to be totally bounded (by some authors: pre-compact) if for every non-empty open subset $U$ of $G$ there is a finite subset $F$ of $G$ such that $G=F U$. It is clear that a compact topological group, and each of its subgroups, is totally bounded. It is a theorem of Weil [27] that, conversely, every totally bounded topological group $G$ is a dense subgroup of a compact group $\bar{G}$. (The two natural uniform structures on such a group $G$ are identical; as a space, $\bar{G}$ may be realized as the completion of $G$ with respect to this uniformity.) The compact group $\bar{G}$ is unique in the sense that if $K$ is a compact topological group in which $G$ is dense then there is a topological isomorphism $\zeta$ from $\bar{G}$ onto $K$ such that $\zeta(x)=x$ for all $x \in G$. We refer to $\bar{G}$ as the Weil completion of the (totally bounded) topological group $G$.

In what follows we denote by $\mathscr{B}(G)$ the set of totally bounded topological group topologies for the group $G$. That is, we write $\mathscr{T} \in \mathscr{B}(G)$ if and only if $\langle G, \mathscr{T}\rangle$ is a totally bounded topological group.

For an Abelian topological group $G=\langle G, \mathscr{T}\rangle$, we denote by $\hat{G}$ or by $\langle G, \mathscr{T}\rangle^{\wedge}$ the set of continuous homomorphisms from $G$ to $\mathbf{T}$. It is well-known (see for example [13] $(22.12,22.17)$ ) that $\hat{G}$ separates points of $G$ whenever $G$ is compact (hence also, whenever $G$ is totally bounded).

For $G$ an Abelian group and $I$ a point-separating subgroup of $\operatorname{Hom}(G, \mathscr{T})$, we denote by $\mathscr{T}_{I}$ the topology induced on $G$ by $I$. The following result establishes an order-isomorphism between $\mathscr{B}(G)$ and the set of point-separating subgroups of $\operatorname{Hom}(G, \mathscr{T})$.

4.1. Theorem [6]. Let $G$ be an Abelian group and let $I, J$ be point-separating subgroups of $\operatorname{Hom}(G, T)$. Then

(a) $I=J$ if and only if $\mathscr{T}_{I}=\mathscr{T}_{J}$;

(b) $\mathscr{T}_{I} \in \mathscr{B}(G)$; and

(c) if $\mathscr{T} \in \mathscr{B}(G)$ and $I=\langle G, \mathscr{T}\rangle$, then $\mathscr{T}=\mathscr{T}_{I}$.

For a topological space $X=\langle X, \mathscr{T}\rangle$ we de.ıne the density character $d(X)$, the weight $w(X)$, the local character $\chi(x, X)$ at $x$, the character $\chi(X)$, and the compact-covering number $\kappa(X)$, by the relations

$d(X)=\min \{|A|: A \subset X, A$ dense in $X\}$,

$w(X)=\min \{|\mathscr{A}|: \mathscr{A} \subset \mathscr{T}, \mathscr{A}$ is a base $\}$, 
$\chi(x, X)=\min \{|\mathscr{A}|: \mathscr{A} \subset \mathscr{T}, \mathscr{A}$ is a local base at $x\}$,

$\chi(X)=\sup \{\chi(x, X): x \in X\}$, and

$\kappa(X)=\min \{|\mathscr{K}|: \mathscr{K} \subset \mathscr{P}(X), X=\bigcup \mathscr{K}$, each $F \in \mathscr{K}$ is compact $\}$.

Of course when $X$ is homogeneous the function $x \rightarrow \chi(x, X)$ is constant. In particular, for a topological group $G$ we have $\chi(G)=\chi(e, G)$.

Here in 4.2-4.4 we list those results concerning these cardinal invariants which we need below, together with references which the interested reader may consult for proofs, generalizations, or related results.

4.2. THEOREM. Let $G$ be an infinite compact topological group. Then

(a) $|G|=2^{w(G)}$;

(b) $d(G)=\log (w(G))$.

Proof. Kakutani [18]; Kuz'minov [21]; Itzkowitz [16]; Hewitt and Ross [14] (28.2, 28.58); Comfort [5].

4.3. THEOREM. Let $G=\langle G, \mathscr{T}\rangle$ be an infinite, totally bounded topological group. Then

(a) $\chi(G)=w(G)$.

(b) If in addition $G$ is Abelian, then $\chi(G)=|\hat{G}|$.

Proof. (a) Of course $\chi(G) \leq w(G)$. Now let $\left\{U_{\xi}: \xi<\beta\right\}$ be a local base for $\langle G, \mathscr{T}\rangle$ at $e$, and for $\xi<\beta$ choose $\mathscr{T}$-open $V_{\xi}$ such that $e \in V_{\xi} \subset$ $V_{\xi}^{-1} V_{\xi} \subset U_{\xi}$ and choose finite $F_{\xi} \subset G$ such that $G=F_{\xi} \cdot V_{\xi}$. For every (basic) neighborhood $p U_{\xi}$ of $p \in G$ there is $x \in F_{\xi}$ such that

$$
p \in x V_{\xi} \subset p V_{\xi}^{-1} V_{\xi} \subset p_{\xi} U_{\xi} \text {. }
$$

It follows that the family $\left\{x V_{\xi}: \xi<\beta, x \in F_{\xi}\right\}$, whose cardinality is $\beta \cdot \omega=\beta$, is a base for $\langle G, \mathscr{T}\rangle$. Hence $w(G) \leq \chi(G)$.

(b) Since $\hat{G}$ induces on $G$ the topology $\mathscr{T}$, the function $i: G \rightarrow \mathbf{T}^{\hat{G}}$ defined by $i(x)_{f}=f(x)$ is a homeomorphism of $\langle G, \mathscr{T}\rangle$ into $\mathbf{T}^{\hat{G}}$. Hence $w(G, \mathscr{T}) \leq w\left(\mathbf{T}^{\hat{G}}\right)=|\hat{G}|$. Suppose now that $\chi(G)=\beta<|\hat{G}|$ and let $\left\{U\left(f_{\xi}, \varepsilon_{\xi}\right): \xi<\beta\right\}$ be a local base at $e$ of sets of the form

$$
U\left(f_{\xi}, \varepsilon_{\xi}\right)=\left\{x \in G:\left|f_{\xi}(x)-1\right|<\varepsilon_{\xi}\right\}
$$

with $f_{\xi} \in \hat{G}, \varepsilon_{\xi}>0$. Let $I$ be the subgroup of $\hat{G}$ generated by $\left\{f_{\xi}: \xi<\beta\right\}$. From $\beta \geq \omega$ we have $|I|=\beta$, but since $I$ itself induces on $G$ the topology $\mathscr{T}$ we have $I=\hat{G}$ from $4.1(\mathrm{c})$. This contradiction shows $\chi(G) \geq|\hat{G}|$, as required.

(We note in passing that each of the three functions $\chi, w, \hat{\mid}$ assumes the same value on $G$ as on $\bar{G}$, so that 4.3 as stated follows from the special 
case in which $G$ is assumed compact. In this case $\hat{G}$ is discrete in the Pontrjagin topology, so that $|\hat{G}|=\kappa(\hat{G})$. The relation $\chi(G)=\kappa(\hat{G})$, from which 4.3(b) now follows, is given for locally compact Abelian groups by Hewitt and Stromberg [15] (the case $\kappa=\omega$ ), by Hewitt and Ross [13] (24.48), and Comfort [5] (3.14(ii)).)

4.4. TheOREM. Let $G$ be an Abelian group with $|G|=\alpha \geq \omega$. Then there is a point-separating subgroup $S$ of $\operatorname{Hom}(G, \mathbf{T})$ such that $|S|=\log (\alpha)$.

Proof. Let $G$ have the discrete topology, so that $\hat{G}$ is compact (Pontrjagin topology). Applying 4.3 and 4.2(b) to $\hat{G}$, we have

$$
w(\hat{G})=|\hat{\hat{G}}|=|G|=\alpha
$$

and then $d(\hat{G})=\log (\alpha)$. Let $D$ be dense in $\hat{G}$ with $|D|=\log (\alpha)$ and let $S$ be the subgroup of $\operatorname{Hom}(G, \mathbf{T})$ generated by $D$. Then $|S|=\log (\alpha)$, and $S$ separates points: If $e \neq x \in G$ there is $h \in \operatorname{Hom}(G, \mathbf{T})$ such that

$$
|h(x)-h(e)|=|h(x)-1|=\varepsilon>0,
$$

and since (with $G$ discrete) the Pontrjagin topology of $\hat{G}$ is the topology of pointwise convergence there is $f \in S$ such that $|f(x)-h(x)|<\varepsilon$ and hence

$$
|f(x)-f(e)|=|f(x)-1| \geq|h(x)-1|-|f(x)-h(x)|>0 .
$$

For use in 5.4 and 5.7 we note in 4.6 a refinement of Theorem 4.4.

We recall that $\mathbf{W}$ denotes $\oplus_{p} \mathbf{Z}\left(p^{\infty}\right)$, the torsion subgroup of $\mathbf{T}$.

4.5. LEMMA. There is a countable, point-separating subset $F$ of $\operatorname{Hom}(\mathbf{T}, \mathbf{W})$.

Proof. Since $\mathbf{W}$ is divisible and $|\mathbf{Q}|=\omega$, there is a countable, pointseparating subset $J$ of $\operatorname{Hom}(\mathbf{Q}, \mathbf{W})$. Now it is well-known (see for example [13] (A.14) or [11] (23.1)) that $\mathbf{T}$ is isomorphic to $\mathbf{W} \times \mathbf{Q}^{\omega}$. For

$$
t=\left\langle w,\langle q(n)\rangle_{1 \leq n<\omega}\right\rangle \in \mathbf{W} \times \mathbf{Q}^{\omega}
$$

with $w \in \mathbf{W}$, each $q(n) \in \mathbf{Q}$, define $\pi_{0}(t)=w$ and $\pi_{n}(t)=q(n)$ for $1 \leq n<\omega$. The family

$$
F=\left\{\pi_{0}\right\} \cup\left\{j \circ \pi_{n}: j \in J, 1 \leq n<\omega\right\}
$$

is as required.

4.6. Corollary. Let $G$ be an Abelian group with $|G|=\alpha \geq \omega$. Then there is a point-separating subgroup $S^{\prime}$ of $\operatorname{Hom}(G, \mathbf{W})$ such that $\left|S^{\prime}\right|=\log (\alpha)$. 
Proof. Let $S$ and $F$ be as in 4.4 and 4.5, respectively, with $|S|=\log (\alpha)$ and $|F|=\omega$, and let $S^{\prime}$ be the subgroup of $\operatorname{Hom}(G, \mathbf{W})$ generated by $\{f \circ s: s \in S, f \in F\}$.

5. On the partially ordered set $\mathscr{B}(G)$. For $G$ a group we denote by $\mathscr{B}(G)$, or simply by $\mathscr{B}$ if there is no chance of ambiguity, the set of totally bounded (Hausdorff) topological group topologies for $G$. And for each cardinal $\gamma$ we define $\mathscr{B}(G, \gamma)$, or simply $\mathscr{B}(\gamma)$, by the rule

$$
\mathscr{B}(\gamma)=\{\mathscr{T} \in \mathscr{B}: \chi(G, \mathscr{T})=\gamma\} \text {. }
$$

Clearly $\mathscr{B}=\bigcup\{\mathscr{B}(\gamma): \gamma$ is a cardinal $\}$.

The following statement is available, implicitly or directly, in [6], [9] or [7]. We record it here in passing because it provides some qualitative information about the partially ordered set $\mathscr{B}(G)$; our principal interest, however, is quantitative (see 5.3).

5.1. TheOREM. Let $G$ be an Abelian group and let $\mathscr{C}, \mathscr{D} \subset \mathscr{B}=\mathscr{B}(G)$ with $\mathscr{C} \neq \varnothing$. Then

(a) $\mathscr{C}$ has a least upper bound in $\mathscr{B}$; and

(b) if $\mathscr{D}$ has a lower bound in $\mathscr{B}$ then $\mathscr{D}$ has a greatest lower bound in $\mathscr{B}$.

Proof. For every $\mathscr{T} \in \mathscr{C} \cup \mathscr{D}$ there is (by 4.1 above) $I \in \mathscr{S}(\operatorname{Hom}(G, \mathbf{T}))$ such that $\mathscr{T}=\mathscr{T}_{I}$; and since $\mathscr{D}$ is bounded below there is $M \in$ $\mathscr{S}(\operatorname{Hom}(G, \mathbf{T}))$ such that $M \subset I$ whenever $\mathscr{T}_{I} \in \mathscr{D}$.

Let $J$ be the subgroup of $\operatorname{Hom}(G, \mathbf{T})$ generated by $\bigcup\left\{I: \mathscr{T}_{I} \in \mathscr{C}\right\}$, and set $K=\bigcap\left\{I: \mathscr{T}_{I} \in \mathscr{D}\right\}$. We have $K \supseteq M$, so that $K$ separates points of $G$. It is now clear, with $\mathscr{U}=\mathscr{T}_{J}$ and $\mathscr{V}=\mathscr{T}_{K}$, that $\mathscr{U}, \mathscr{V} \in \mathscr{B}$ and $\mathscr{U}=\mathrm{V} \mathscr{C}$, $\mathscr{V}=\Lambda \mathscr{D}$.

We turn now to computing various cardinal numbers associated with the partially ordered sets $\mathscr{B}(\gamma)$. We show first that for $\gamma$ too small or too large, the sets $\mathscr{B}(\gamma)$ collapse to $\varnothing$.

5.2. THEOREM. Let $G$ be an Abelian group such that $|G|=\alpha \geq \omega$, and let $\gamma \geq \omega$. If $\gamma<\log (\alpha)$ or $\gamma>2^{\alpha}$, then $\mathscr{B}(G, \gamma)=\varnothing$.

Proof. To show the contrapositive, let $\mathscr{T} \in \mathscr{B}(G, \gamma)$ and denote by $\bar{G}$ the Weil completion (defined as in $\S 4$ ) of $\langle G, \mathscr{T}\rangle$. We have from 4.2(a) and 4.3(a) that

$$
\alpha=|G| \leq|\bar{G}|=2^{w(\bar{G})}=2^{x(\bar{G})}=2^{\chi(G)}=2^{\gamma},
$$

so that $\log (\alpha) \leq \gamma$. Further from 4.3(b) and 1.4 we have

$$
\gamma=\chi(G)=\left|\langle G, \mathscr{T}\rangle^{\wedge}\right| \leq|\operatorname{Hom}(G, \mathbf{T})|=2^{\alpha} .
$$

The proof is complete. 
(Alternatively, the inequality $\gamma \leq 2^{\alpha}$ may be derived from an argument which does not invoke $\operatorname{Hom}(G, T)$. See 6.2(a) below.)

As in $\$ 1$, the expressions "anti-chain", "well-ordered set" and "antiwell-ordered set", when applied to families of subsets of a fixed set, refer to the partial order given by set-theoretic inclusion. We introduce now the following (quite standard) terminology.

DEFINITION. Let $P$ be a partially ordered set. Then $\operatorname{width}(P)=\sup \{|A|: A \subset P, A$ is an anti-chain $\}$; $\operatorname{height}(P)=\sup \{|W|: W \subset P, W$ is well-ordered $\}$; and $\operatorname{depth}(P)=\sup \left\{\left|W^{*}\right|: W^{*} \subset P, W^{*}\right.$ is anti-well-ordered $\}$.

5.3. THEOREM. Let $G$ be an Abelian group with $|G|=\alpha \geq \omega$, and let $\log (\alpha) \leq \gamma \leq 2^{\alpha}$. Define $\bar{\gamma}=\min \left\{\gamma^{+}, 2^{\alpha}\right\}$. Then

(a) $|\mathscr{B}(\gamma)|=2^{\alpha \cdot \gamma}$.

(b) The width of $\mathscr{B}(\gamma)$ is $2^{\alpha \cdot \gamma}$ and there is an anti-chain $\mathscr{A} \subset \mathscr{B}(\gamma)$ such that $|\mathscr{A}|=2^{\alpha \cdot \gamma}$.

(c) The height of $\mathscr{B}(\gamma)$ is $\bar{\gamma}$ and there is a well-ordered $\mathscr{W} \subset \mathscr{B}(\gamma)$ such that $|\mathscr{W}|=\bar{\gamma}$.

(d) The depth of $\mathscr{B}(\gamma)$ is $\gamma$ and there is an anti-well-ordered $\mathscr{W}^{*} \subset \mathscr{B}(\gamma)$ such that $\left|\mathscr{W}^{*}\right|=\gamma$.

Proof. Throughout this proof we write $H=\operatorname{Hom}(G, \mathbf{T})$.

According to 4.1 and 4.3(b), the elements of $\mathscr{B}(\gamma)$ are in one-to-one order-preserving correspondence with the point-separating elements of $\mathscr{S}(H, \gamma)$. From this follows easily the equality $|\mathscr{B}(\gamma)| \leq 2^{\alpha \cdot \gamma}$ of (a), together with the fact that width $\mathscr{B}(\gamma))$, height $(\mathscr{B}(\gamma))$, and depth $(\mathscr{B}(\gamma))$ are bounded above respectively by $2^{\alpha \cdot \gamma}, \bar{\gamma}$ and $\gamma$. (In detail: We have $|H|=2^{\alpha}$, and a set of cardinality $2^{\alpha}$ has exactly $2^{\alpha \cdot \gamma}$ subsets of cardinality $\gamma$ if $1 \leq \gamma \leq 2^{\alpha}$.) Thus

$$
|\mathscr{B}(\gamma)| \leq|\mathscr{S}(H, \gamma)| \leq 2^{\alpha \cdot \gamma} .
$$

The inequality width $(\mathscr{B}(\gamma)) \leq|\mathscr{B}(\gamma)|$ is clear from the definition. Suppose now that $\left\{U_{\xi}: \xi<\sigma\right\}$ and $\left\{\mathscr{V}_{\eta}: \eta<\tau\right\}$ are subsets of $\mathscr{B}(\gamma)$ such that the functions $\xi \rightarrow \mathscr{U}_{\xi}$ and $\eta \rightarrow \mathscr{V}_{\eta}$ are an isomorphism and an anti-isomorphism respectively from $\sigma$ and $\tau$ into $\mathscr{B}(\gamma)$, and for $\xi<\sigma, \eta<\tau$ set $I_{\xi}=\left\langle G, \mathscr{U}_{\xi}\right\rangle$ and $J_{\eta}=\left\langle G, \mathscr{V}_{\eta}\right\rangle$. Then the functions $\xi \rightarrow I_{\xi}, \eta \rightarrow J_{\eta}$ are an isomorphism and an anti-isomorphism respectively from $\sigma$ and $\tau$ into $\mathscr{S}(H, \gamma)$. If $\sigma>\gamma^{+}$then since $I_{\xi+1} \backslash I_{\xi} \neq \varnothing$ for $\xi<\gamma^{+}$we have $\left|I_{\gamma^{+}}\right| \geq \gamma^{+}$; 
this contradiction shows $\sigma \leq \gamma^{+}$. The inequality $\sigma \leq|H|=2^{\alpha}$ being obvious, we conclude that $\sigma \leq \bar{\gamma}$. Finally since $J_{\eta} \backslash J_{\eta+1} \neq \varnothing$ for $\eta<\tau$ and $\left|J_{0}\right|=\gamma$, we have $\tau \leq \gamma$.

Since the inequality $\geq$ of (a) follows from (b), to complete the proof it is enough to show that the upper bounds $2^{\alpha \cdot \gamma}, \bar{\gamma}$ and $\gamma$ are assumed as asserted in (b), (c) and (d) respectively.

We have from 4.4 that there is $S \in \mathscr{S}(H)$ such that $S$ separates points of $G$ and

$$
|S|=\log (\alpha) \leq \alpha<2^{\alpha} ;
$$

clearly we have $|H / S|=2^{\alpha}>\omega$. It then follows from 1.3 , with $G$ and $\alpha$ replaced by $H / S$ and $2^{\alpha}$, respectively, that there are $\mathscr{A}(H / S), \mathscr{W}(H / S)$, $\mathscr{W}^{*}(H / S) \subset \mathscr{S}(H / S, \gamma)$ such that

(i) $|\mathscr{A}(H / S)|=2^{\alpha \cdot \gamma}$ and the elements of $\mathscr{A}(H / S)$ are pairwise incomparable;

(ii) $|\mathscr{W}(H / S)|=\bar{\gamma}$ and $\mathscr{W}(H / S)$ is well-ordered; and

(iii) $\left|\mathscr{W}^{*}(H / S)\right|=\gamma$ and $\mathscr{W}^{*}(H / S)$ is anti-well-ordered.

For every $A \in \mathscr{S}(H / S)$ there is $\tilde{A} \in \mathscr{S}(H)$ such that $A=\tilde{A} / S$. We define

$$
\begin{aligned}
\mathscr{A}(H) & =\{\tilde{A}: A \in \mathscr{A}(H / S)\}, \\
\mathscr{W}(H) & =\{\tilde{A}: A \in \mathscr{W}(H / S)\}, \text { and } \\
\mathscr{W} *(H) & =\left\{\tilde{A}: A \in \mathscr{W}^{*}(H / S)\right\} .
\end{aligned}
$$

We note that for $A \in \mathscr{S}(H / S, \gamma)$ we have $|\tilde{A}|=\gamma \cdot|S|=\gamma$; thus $\mathscr{A}(H)$, $\mathscr{W}(H), \mathscr{W}^{*}(H) \subset \mathscr{S}(H, \gamma)$. Further, these three families retain the cardinality and set-theoretic properties given in (i), (ii) and (iii), respectively.

Finally we set

$$
\begin{aligned}
\mathscr{A} & =\left\{\mathscr{T}_{\tilde{A}}: \tilde{A} \in \mathscr{A}(H)\right\}, \\
\mathscr{W} & =\left\{\mathscr{T}_{\tilde{A}}: \tilde{A} \in \mathscr{W}(H)\right\}, \quad \text { and } \\
\mathscr{W}^{*} & =\left\{\mathscr{T}_{\tilde{A}}: \tilde{A} \in \mathscr{W}^{*}(H)\right\} .
\end{aligned}
$$

It follows from 4.1 that the families $\mathscr{A}, \mathscr{W}$ and $\mathscr{W}^{*}$ are as required in (b), (c) and (d) respectively.

The proof is complete.

In 5.4-5.8, we offer some comments and corollaries deriving from 5.3. 5.4. (Here we pursue a remark suggested in conversation by Lewis $C$. Robertson.) All of the totally bounded topologies considered and described in Theorem 5.3 may be chosen to be totally disconnected in the sense that 
no connected subset has more than one point. We now describe how to alter the proof of 5.3 , using 4.6 , so as to show this.

Given $G$ and $\gamma$ as in the statement of 5.3, there is by 4.6 a point-separating subgroup $S^{\prime}$ of $\operatorname{Hom}(G, \mathbf{W})$ such that $\left|S^{\prime}\right|=\log (\alpha)$. For $s \in S^{\prime}$, the group $s[G]$ is a countable subgroup of $\mathbf{T}$, hence is totally disconnected (indeed, zero-dimensional in the sense that there is a basis of open-andclosed sets). The group $\Pi_{s \in S^{\prime}} s[G]$ is then also totally disconnected, and hence the topology $\mathscr{T}_{S^{\prime}}$ on $G$, which is characterized by the property that the embedding $i: G \rightarrow \prod_{s \in S^{\prime}} s[G]$ defined by $i(x)_{s}=s(x)$ is a homeomorphism, is totally disconnected (indeed, zero-dimensional).

In the proof of Theorem 5.3, all of the topologies constructed (in $\mathscr{A}$, $\mathscr{W}$ and $\left.\mathscr{W}^{*}\right)$ are of the form $\mathscr{T}_{\tilde{A}}$ with $S \subset \tilde{A} \in \mathscr{S}(H), H=\operatorname{Hom}(G, \mathbf{T})$. For present purposes it is enough throughout that argument to replace $S$ by $S^{\prime}$ as above; we have $S^{\prime} \in \mathscr{S}(\operatorname{Hom}(G, \mathrm{~W})) \subset \mathscr{S}(H)$. Since the topologies $\mathscr{T}_{\tilde{A}}$ so defined on $G$ contain the totally disconnected topology $\mathscr{T}_{S^{\prime}}$, each such topology $\mathscr{T}_{\tilde{A}}$ is itself totally disconnected.

Let $\mathscr{B}^{\prime}(G, \gamma)$ denote the set of totally bounded, totally disconnected topological group topologies $\mathscr{T}$ on $G$ such that $\chi(G, \mathscr{T})=\gamma$. The upshot of the preceding two paragraphs is that (b), (c) and (d) of 5.3 can be strengthened to $\left(b^{\prime}\right),\left(c^{\prime}\right)$ and $\left(d^{\prime}\right)$. We state $\left(b^{\prime}\right)$ only, leaving $\left(c^{\prime}\right)$ and $\left(d^{\prime}\right)$ to the reader.

(b') The width of $\mathscr{B}(G, \gamma)$ is $2^{\alpha \cdot \gamma}$ and there is an anti-chain $\mathscr{A} \subset$ $\mathscr{B}^{\prime}(G, \gamma)$ such that $|\mathscr{A}|=2^{\alpha \cdot \gamma}$.

5.5. If $2^{\gamma}>2^{\alpha}$ (in particular, if $\gamma=2^{\alpha}$ ), then the family $\mathscr{A}$ of $5.3(\mathrm{~b})$ may be chosen so that $|\mathscr{A}|=2^{\alpha \cdot \gamma}=2^{\gamma}$ and the topologies in $\mathscr{A}$ are both non-comparable and non-homeomorphic. (The following simple argument applies in a very broad context.) For $\mathscr{T}, \mathscr{U} \in \mathscr{A}$ write $\mathscr{T} \sim \mathscr{U}$ if the spaces $\langle G, \mathscr{T}\rangle$ and $\langle G, \mathscr{U}\rangle$ are homeomorphic, and for $\mathscr{T} \in \mathscr{A}$ set

$$
E(\mathscr{T})=\{\mathscr{U} \in \mathscr{A}: \mathscr{T} \sim \mathscr{U}\} .
$$

A homeomorphism of the kind here considered is, among other things, a function from $G$ to $G$. Since there are only $2^{\alpha}$ such functions, we have $|E(\mathscr{T})| \leq 2^{\alpha}$ for each $\mathscr{T} \in \mathscr{A}$. Since $|\mathscr{A}|=2^{\gamma}>2^{\alpha}$ there are $2^{\gamma}$ distinct classes of the form $E(\mathscr{T})$; it is then immediate that there is $\mathscr{A}^{*} \subset \mathscr{A}$ such that $\left|\mathscr{A}^{*}\right|=2^{\gamma}$ and the elements of $\mathscr{A}^{*}$ are pairwise non-homeomorphic.

5.6. When $\gamma=2^{\alpha}$ we have $2^{\alpha \cdot \gamma}=2^{2^{\alpha}}$ and $\bar{\gamma}=2^{\alpha}$. Since $\mathscr{B}=\mathscr{B}(G) \subset$ $\mathscr{P}\left(\mathscr{P}(G)\right.$ ) we have $|\mathscr{B}| \leq 2^{2^{\alpha}}$, and Theorem 5.3 (together with 5.4 and 5.5) yields the following information concerning the set $\mathscr{B}=\mathscr{B}(G)$ of all totally bounded topological group topologies on $G$ and the set $\mathscr{B}^{\prime}$ of totally disconnected topologies in $\mathscr{B}$. 
5.7. Corollary. Let $G$ be an Abelian group with $|G|=\alpha \geq \omega$. Then

(a) $|\mathscr{B}|=2^{2^{\alpha}}$;

(b) $\operatorname{width}(\mathscr{B})=2^{2^{\alpha}}$ and there is an anti-chain $\mathscr{A} \subset \mathscr{B}^{\prime}$ such that $|\mathscr{A}|=$ $2^{2^{\alpha}}$ and the elements of $\mathscr{A}$ are pairwise non-homeomorphic;

(c) height $(\mathscr{B})=2^{\alpha}$ and there is well-ordered $\mathscr{W} \subset \mathscr{B}^{\prime}$ such that $|\mathscr{W}|=$ $2^{\alpha} ;$ and

(d) $\operatorname{depth}(\mathscr{B})=2^{\alpha}$ and there is anti-well-ordered $\mathscr{W}^{*} \subset \mathscr{B}^{\prime}$ such that $\mid$ are $* \mid=2^{\alpha}$.

5.8. The present paper originated with the authors' desire, given an infinite Abelian group $G$, to determine (among all topological group topologies for $G$ ) the number which are totally bounded, the number which are metrizable, and the number which are both totally bounded and metrizable. Corollary 5.6(a) responds to the first of these, and 6.2 below is devoted to (generalizations of) the second. As to the third, let us recall first a non-trivial result proved independently by Kakutani [17] and Birkhoff [4], recorded also by Hewitt and Ross [13] (Theorem 8.3): A topological group $G$ is metrizable if and only if $\chi(G) \leq \omega$-that is, if and only if $G$ is first countable. In the notation introduced above, then, the totally bounded metrizable topological group topologies for an infinite Abelian group $G$ are exactly the elements of $\mathscr{B}(G, \omega)$. Theorem 5.2 and 5.3(a) for $\gamma=\omega$ reduce to the statements that $\mathscr{B}(G, \omega)=\varnothing$ if $\alpha>2^{\omega}$, and $|\mathscr{B}(G, \omega)|=2^{\alpha}$ if $\omega \leq \alpha \leq 2^{\omega}$. We leave to the interested reader the task of interpreting the statements of 5.3(b), (c), (d) and 5.4 concerning width, height and depth into this (metrizable) context.

6. How many metrizable topologies? Given an (infinite) Abelian group $G$, we determine here the number of metrizable topological group topologies which $G$ admits. The theorem cited above of Kakutani [17] and Birkhoff [4], to the effect that $\langle G, \mathscr{T}\rangle$ is metrizable if and only if $\chi(G, \mathscr{T}) \leq \omega$, suggests as a natural generalization the project of counting, for fixed $\gamma \geq \omega$, the number of $\mathscr{T}$ for which $\chi(G, \mathscr{T})=\gamma$. This we achieve in Theorem 6.2 below. The following notation is helpful.

Definition. Let $G$ be a group and let $\gamma \geq \omega$. The set $\mathscr{M}(G, \gamma)$, also denoted $\mathscr{M}(\gamma)$ when confusion is impossible, is the set of topological group topologies $\mathscr{T}$ for $G$ such that $\chi(G, \mathscr{T})=\gamma$.

In the notation of $\S 5$ above we have

$$
\mathscr{B}(G, \gamma)=\mathscr{M}(G, \gamma) \cap \mathscr{B}(G)
$$

for all groups $G$ and all cardinals $\gamma \geq \omega$. 
We begin by showing that under appropriate circumstances a topology $\mathscr{T}$ in $\mathscr{M}(G, \gamma)$ can be chosen with a local basis at $e$ of subgroups.

6.1. Lemma. Let $\gamma \geq \omega$ and let $G$ be an Abelian group of the form $G=\oplus_{i \in I} G_{i}$ with $|I|=\gamma$ and with $2 \leq\left|G_{i}\right| \leq \omega$ for each $i \in I$. Then there is a topological group topology $\mathscr{T}$ for $G$ such that $\chi(G, \mathscr{T})=\gamma$ and $\langle G, \mathscr{T}\rangle$ has a local base at e whose elements are subgroups of $G$.

Proof. If each $G_{i}$ is given the discrete topology, the topology $\mathscr{T}$ induced on $G$ by the product topology of $\prod_{i \in I} G_{i}$ is as required. Indeed for finite $F \subset I$ set

$$
H(F)=\left\{x \in G: i \in F \text { implies } x_{i}=e_{i}\right\} .
$$

It is then clear (and it follows also from [13] (4.5, 4.21(a))) that the family

$$
\mathscr{A}=\{H(F): \text { finite } F \subset I\}
$$

has the property that $\{x+H(F): x \in G, H(F) \in \mathscr{A}\}$ is a base for $\mathscr{T}$ and $\langle G, \mathscr{T}\rangle$ is a topological group. From $|\mathscr{A}|=\gamma$ we have $\chi(G, \mathscr{T}) \leq \gamma$. If $\mathscr{A}^{\prime} \subset \mathscr{A}$ with $\left|\mathscr{A}^{\prime}\right|<\gamma$ there is

$$
i \in I \backslash \bigcup\left\{F: H(F) \in \mathscr{A}^{\prime}\right\},
$$

so since $\left|G_{i}\right| \geq 2$ there is $x \in \bigcap \mathscr{A}^{\prime}$ such that $x \neq e$ (indeed, with $x_{i} \neq e_{i}$ ). This shows that $\{e\}$ is not the intersection of fewer than $\gamma$ many elements of $\mathscr{A}$. It follows that $\chi(G, \mathscr{T}) \geq \gamma$, as required.

We turn now to the computation of $|\mathscr{M}(G, \gamma)|$.

6.2. ThEOREM. Let $\alpha$ and $\gamma$ be infinite cardinal numbers and let $G$ be an Abelian group such that $|G|=\alpha$. Then

(a) $\mathscr{M}(G, \gamma)=\varnothing$ if $\gamma>2^{\alpha}$

(b) $|\mathscr{M}(G, \gamma)|=2^{\alpha \cdot \gamma}$ if $\log (\alpha) \leq \gamma \leq 2^{\alpha}$; and

(c) $|\mathscr{M}(G, \gamma)|=2^{\alpha}$ if $\omega \leq \gamma<\log (\alpha)$.

Proof. Throughout this proof we write $\mathscr{M}(\gamma)=\mathscr{M}(G, \gamma)$.

Set $\Phi=\{\mathscr{F} \subset \mathscr{P}(G):|\mathscr{F}|=\gamma\}$. For every $\mathscr{T} \in \mathscr{M}(\gamma)$ there is $\mathscr{F}(\mathscr{T})$ $\in \Phi$ such that $\mathscr{F}(\mathscr{T})$ is a base at $e$ for $\langle G, \mathscr{T}\rangle$. The function $\mathscr{T} \rightarrow \mathscr{F}(\mathscr{T})$ is one-to-one from $\mathscr{M}(\gamma)$ into $\Phi$. For $\gamma>2^{\alpha}$ we have $\Phi=\varnothing$ and hence $\mathscr{M}(\gamma)=\varnothing$; this is (a). For $1 \leq \gamma \leq 2^{\alpha}$ we have $|\Phi|=\left(2^{\alpha}\right)^{\gamma}$ and hence

$$
|\mathscr{M}(\gamma)| \leq 2^{\alpha \cdot \gamma} \text {. }
$$


When $\log (\alpha) \leq \gamma \leq 2^{\alpha}$, relation $(*)$ and the equality $\mathscr{B}(\gamma)=\mathscr{M}(\gamma) \cap \mathscr{B}$ and Theorem 5.3(a) combine to yield

$$
2^{\alpha \cdot \gamma}=|\mathscr{B}(\gamma)|=|\mathscr{M}(\gamma) \cap \mathscr{B}| \leq|\mathscr{M}(\gamma)| \leq 2^{\alpha \cdot \gamma},
$$

which is (b). And when $\omega \leq \gamma<\log (\alpha)$ (indeed, when $\omega \leq \gamma \leq \alpha$ ), relation (*) becomes $|\mathscr{M}(\gamma)| \leq 2^{\alpha}$. Thus to complete the proof it is enough to verify the inequality $\geq$ of (c); the following argument is valid for all cases $\omega \leq \gamma \leq \alpha$ with $\alpha>\omega$.

To show $|\mathscr{M}(\gamma)| \geq 2^{\alpha}$ it is clearly enough to show there is a subgroup $H$ of $G$ such that $|\mathscr{M}(H, \gamma)| \geq 2^{\alpha}$. (Indeed for $\mathscr{U} \in \mathscr{M}(H, \gamma)$ the topology $\mathscr{T}(\mathscr{U})$ for $G$ generated by the requirement that $\langle H, \mathscr{U}\rangle$ is an open topological subgroup of $\langle G, \mathscr{T}(\mathscr{U})\rangle$ satisfies $\mathscr{T}(\mathscr{U}) \in \mathscr{M}(\gamma)$, and the function $\mathscr{U} \rightarrow \mathscr{T}(\mathscr{U})$ is one-to-one from $\mathscr{M}(H, \gamma)$ into $\mathscr{M}(\gamma)$.) Thus we assume without loss of generality, using 1.1, that $G$ has the form $G=$ $\oplus_{\eta<\alpha} G_{\eta}^{\prime}$ with $2 \leq\left|G_{\eta}^{\prime}\right| \leq \omega$ for all $\eta<\alpha$. Choosing a family $\left\{A_{\xi}: \xi<\alpha\right\}$ of pairwise disjoint subsets of $\alpha$ such that each $\left|A_{\xi}\right|=\gamma$ and $\bigcup_{\xi<\alpha} A_{\xi}=\alpha$, and writing $G_{\xi}=\bigoplus_{\eta \in A_{\xi}} G_{\eta}^{\prime}$, we have $G=\oplus_{\xi<\alpha} G_{\xi}$; further, each group

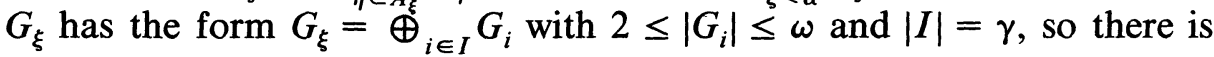
by Lemma 6.1 for $\xi<\alpha$ an element $\mathscr{T}_{\xi}$ of $\mathscr{M}\left(G_{\xi}, \gamma\right)$ such that $\left\langle G_{\xi}, \mathscr{T}_{\xi}\right\rangle$ has a local base $\left\{H_{\xi}(\eta): \eta<\gamma\right\}$ at $e_{\xi}$ whose elements are subgroups of $G_{\xi}$.

Now let $\varnothing \neq A \subsetneq \alpha$ and set $G_{A}=\oplus_{\xi \in A} G_{\xi}$. We will associate with $A$ an element $\mathscr{T}(A)$ of $\mathscr{M}(\gamma)$ (and we will show that the function $A \rightarrow \mathscr{T}(A)$ is one-to-one).

We note that $G=G_{A} \times G_{\alpha \backslash A}$. To define $\mathscr{T}(A)$ we will define $\mathscr{U}(A)$ $\in \mathscr{M}\left(G_{A}, \gamma\right)$, we will denote by $\mathscr{V}(A)$ the discrete topology for $G_{\alpha \backslash A}$, and we will define $\langle G, \mathscr{T}(A)\rangle$ to be the product of the two topological groups $\left\langle G_{A}, \mathscr{U}(A)\right\rangle$ and $\left\langle G_{\alpha \backslash A}, \mathscr{V}(A)\right\rangle$.

We set $H_{A}(\eta)=\oplus_{\xi \in A} H_{\xi}(\eta)$ for $\eta<\gamma$, and we define $\mathscr{U}(A)$ by the requirement that $\left\{H_{A}(\eta): \eta<\gamma\right\}$ is a local neighborhood base at $e_{A}$. (In detail: A subset $U$ of $G_{A}$ is $\mathscr{U}(A)$-open if and only if for each $x \in U$ there is $\eta<\gamma$ such that $x+H_{A}(\eta) \subset U$.) As in the proof of 6.1 , that $\mathscr{U}(A)$ is a (Hausdorff) topological group topology for $G_{A}$ may be seen directly or by appeal to [13] (4.5 and 4.2 (a)). Further it is clear, given $\varnothing \neq A \subsetneq \alpha$ and choosing $\xi \in A$, that $\left\langle G_{\xi}, \mathscr{T}_{\xi}\right\rangle$ is (naturally homeomorphic with) a topological subgroup of $\left\langle G_{A}, \mathscr{U}(A)\right\rangle$; this shows

$$
\gamma=\chi\left(G_{\xi}, \mathscr{T}_{\xi}\right) \leq \chi\left(G_{A}, \mathscr{U}(A)\right) \leq\left|\left\{H_{A}(\eta): \eta<\gamma\right\}\right|=\gamma,
$$

so that $\chi\left(G_{A}, \mathscr{U}(A)\right)=\gamma$. It then follows, with $\mathscr{V}(A)$ and $\mathscr{T}(A)$ defined as indicated above, that $\chi(G, \mathscr{T}(A))=\gamma$, i.e., $\mathscr{T}(A) \in \mathscr{M}(\gamma)$.

It remains to show that if $A$ and $A^{*}$ are different non-empty, proper subsets of $\alpha$ then $\mathscr{T}(A) \neq \mathscr{T}\left(A^{*}\right)$. We assume without loss of generality 
that there is $\zeta \in A \backslash A^{*}$ and we define a net $p=\langle p(\eta): \eta\langle\gamma\rangle$ in $G$ by choosing $p(\eta)_{\xi} \in G_{\xi}$ so that

$$
\begin{aligned}
p(\eta)_{\xi}=e_{\xi} & \text { if } \xi \neq \zeta \\
p(\eta)_{\zeta} \in H_{\zeta}(\eta), & p(\eta)_{\zeta} \neq e_{\zeta} .
\end{aligned}
$$

(The set which indexes the net $p=\langle p(\eta)\rangle$ is the set $D=\{\eta: \eta<\gamma\} ; D$ is directed by the relation $\prec$ defined by the rule $\eta \prec \eta^{\prime}$ if $H_{\zeta}\left(\eta^{\prime}\right) \subset H_{\zeta}(\eta)$.) We write

$$
\begin{aligned}
& p(\eta)=\langle q(\eta), r(\eta)\rangle \in G_{A} \times G_{\alpha \backslash A}, \quad \text { and } \\
& p(\eta)=\langle s(\eta), t(\eta)\rangle \in G_{A^{*}} \times G_{\alpha \backslash A^{*}} .
\end{aligned}
$$

It is clear that for every basic $\mathscr{U}(A)$-neighborhood $H_{A}(\eta)$ of $e_{A}$ we have $q\left(\eta^{\prime}\right) \in H_{A}(\eta)$ whenever $\eta \prec \eta^{\prime}$; further, $r\left(\eta^{\prime}\right)=e_{\alpha \backslash A}$. This shows that $p(\eta) \rightarrow e=e_{G}$ in the topology $\mathscr{T}(A)$. But $\left\{e_{\alpha \backslash A^{*}}\right\}$ is a $\mathscr{V}\left(A^{*}\right)$-neighborhood of $e_{\alpha \backslash A^{*}}$, and $t(\eta) \notin\left\{e_{\alpha \backslash A^{*}}\right\}$ since

$$
t(\eta)_{\zeta}=p(\eta)_{\zeta} \neq e_{\zeta} \text { and } \zeta \in \alpha \backslash A^{*} .
$$

Thus $t(\eta) \nrightarrow e_{\alpha \backslash A^{*}}$ in the (discrete) topology $\mathscr{V}\left(A^{*}\right)$ on $G_{\alpha \backslash A^{*}}$, so $p(\eta) \nrightarrow$ $e=e_{G}$ in the topology $\mathscr{T}\left(A^{*}\right)$.

The proof is complete.

6.3. Much as in 5.3, the topologies of Theorem 6.2 may be (indeed, are) chosen to be totally disconnected. In fact for $\varnothing \neq A \subset \alpha$ the family $\left\{H_{A}(\eta): \eta<\gamma\right\}$ is a basis for $\mathscr{U}(A)$ at the identity consisting of openand-closed subgroups. Thus $\langle G, \mathscr{T}(A)\rangle$ is zero-dimensional and, writing

$$
\mathscr{M}^{\prime}(G, \gamma)=\{\mathscr{T} \in \mathscr{M}(G, \gamma):\langle G, \mathscr{T}\rangle \text { is zero-dimensional }\}
$$

we have the following adjunct to 6.2 .

6.4. THEOREM. Let $\alpha$ and $\gamma$ be infinite cardinal numbers and let $G$ be an Abelian group such that $|G|=\alpha$. Then $|\mathscr{M}(G, \gamma)|=\left|\mathscr{M}^{\prime}(G, \gamma)\right|$.

Since the metrizable topological group topologies on $G$ are exactly the elements of $\mathscr{M}(G, \omega)=\mathscr{M}(\omega)$, the case $\gamma=\omega$ of Theorem 6.2 assumes the following form.

6.3. Corollary. Let $G$ be an infinite Abelian group and $\mathscr{M}$ the set of metrizable topological group topologies for $G$. Then $|\mathscr{M}|=2^{|G|}$. 


\section{REFERENCES}

[1] David M. Arnold, Finite Rank Torsion Free Abelian Groups and Rings, Lecture Notes in Mathematics vol. 931, Springer-Verlag, Berlin-Heidelberg-New York, 1982.

[2] R. A. Beaumont and R. S. Pierce, Torsion-free rings, Illinois J. Math., 5 (1961), 61-98.

[3] Shiferaw Berhanu, W. W. Comfort and J. D. Reid, Counting subgroups and topological group topologies, Abstracts Amer. Math. Soc., 4 (1983), 200 [Abstract \# 83T-20-150].

[4] Garrett Birkhoff, A note on topological groups, Compositio Math., 3 (1936), 427-430.

[5] W. W. Comfort, Topological Groups, In: Handbook of General Topology, edited by K. Kunen and J. Vaughan. North-Holland Publ. Co. Amsterdam. 1984. (To appear).

[6] W. W. Comfort and Kenneth A. Ross, Topologies induced by groups of characters, Fundamenta Math., 55 (1964), 283-291.

[7] W. W. Comfort and Victor Saks, Countably compact groups and finest totally bounded topologies, Pacific J. Math., 49 (1973), 33-44.

[8] W. W. Comfort and T. Soundararajan, Pseudocompact group topologies and totally dense subgroups, Pacific J. Math., 100 (1982), 61-84.

[9] J. Dixmier, Les $C^{*}$-algebres et Leurs Représentations, Second edition, Gauthier-Villars, Paris, 1969.

[10] Ryszard Engelking, General Topology, Polska Akademia Nauk, Monographie Matematyczne volume 60, Panstwowe Wydawnictwo Naukowe-Polish Scientific Publishers, Warszawa, 1977.

[11] Laszlo Fuchs, On character groups of discrete Abelian groups, Acta. Math. Acad. Sci. Hungary, 10 (1959), 133-140.

[12] , Infinite Abelian Groups, vol. I. Pure and Applied Mathematics, no. 36. Academic Press. New York and London, 1970.

[13] Edwin Hewitt and Kenneth A. Ross, Abstract Harmonic Analysis, Volume I, Grundlehren der math. Wissenschaften volume 115, Springer-Verlag, Berlin-GöttingenHeidelberg, 1963.

[14] Abstract Harmonic Analysis II, Grundlehren der math. Wissenschaften volume 152, Springer-Verlag, New York-Heidelberg-Berlin, 1970.

[15] Edwin Hewitt and Karl Stromberg, A remark on Fourier-Stieltjes transforms, Anais Academia Brasileira Ciencias, 34 (1962), 174-180.

[16] Gerald L. Itzkowitz, On the density character of compact topological groups, Fundamenta Math., 75 (1972), 201-203.

[17] Shizuo Kakutani, Über die Metrisation der topologischen Gruppen, Proc. Imperial Acad. Tokyo, 12 (1936), 82-84.

[18] _ On cardinal numbers related with a compact Abelian group, Proc. Imperial Acad. Tokyo, 19 (1943), 366-372.

[19] John O. Kiltinen, On the number of field topologies on an infinite field, Proc. Amer. Math. Soc., 40 (1973), 30-36.

[20] _ Infinite Abelian groups are highly topologizable, Duke Math. J., 41 (1974), 151-154.

[21] V. Kuz'minov, On a hypothesis of P.S. Alexandroff in the theory of topological groups, Doklady Akad. Nauk SSSR N. S., 125 (1959), 727-729.

[22] C. Murley, The classification of certain classes of torsion free Abelian groups, Pacific J. Math., 40 (1972), 647-665.

[23] Klaus-Peter Podewski, The number of field topologies on countable fields, Proc. Amer. Math. Soc., 39 (1973), 33-38. 
[24] _ Topologisierung algebraischer Strukturen, Revue Roumaine Math. Pures et Appliquées, 22 (1977), 1283-1290.

[25] J. D. Reid, A note on torsion free Abelian groups of infinite rank, Proc. Amer. Math. Soc., 13 (1962), 222-225.

[26] Dieter Remus, On the structure of the lattice of group topologies, Doctoral Dissertation, Hanover University (Federal Republic of Germany), 1983.

[27] André Weil, Sur les Espaces à Structure Uniforme et sur la Topologie Générale, Publ. Math. Univ. Strasbourg, Hermann \& Cie., Paris, 1937.

Received May 17, 1983. (The third author was supported in part by the National Science Foundation (U.S.A.) under grant NSF-MCS-8004456.)

WESLEYAN UNIVERSITY

Middletown, CT 06457 



\title{
PACIFIC JOURNAL OF MATHEMATICS EDITORS
}

\author{
DONALD BABBITT (Managing Editor) \\ University of California \\ Los Angeles, CA 90024 \\ J. DugundJI \\ University of Southern California \\ Los Angeles, CA 90089-1113 \\ R. FINN \\ Stanford University \\ Stanford, CA 94305 \\ Hermann FlaschKa \\ University of Arizona \\ Tucson, AZ 85721
}

\author{
C. C. MOORE \\ University of California \\ Berkeley, CA 94720 \\ ARTHUR OGUS \\ University of California \\ Berkeley, CA 94720 \\ Hugo Rossi \\ University of Utah \\ Salt Lake City, UT 84112 \\ H. SAMELSON \\ Stanford University \\ Stanford, CA 94305
}

\begin{tabular}{|c|c|c|c|c|}
\hline \multicolumn{5}{|c|}{ ASSOCIATE EDITORS } \\
\hline R. ARENS & $\begin{array}{l}\text { E. F. BECKENBACH } \\
(1906-1982)\end{array}$ & NeumanN & F. Wolf & K. YosHIDA \\
\hline \multicolumn{5}{|c|}{ SUPPORTING INSTITUTIONS } \\
\hline \multirow{2}{*}{\multicolumn{2}{|c|}{$\begin{array}{l}\text { UNIVERSITY OF ARIZONA } \\
\text { UNIVERSITY OF BRITISH COLUMBIA }\end{array}$}} & \multicolumn{3}{|c|}{ UNIVERSITY OF OREGON } \\
\hline & & \multirow{2}{*}{\multicolumn{3}{|c|}{ UNIVERSITY OF SOUTHERN CALIFORNIA }} \\
\hline \multirow{2}{*}{\multicolumn{2}{|c|}{$\begin{array}{l}\text { CALIFORNIA INSTITUTE OF TECHNOLOGY } \\
\text { UNIVERSITY OF CALIFORNIA }\end{array}$}} & & & \\
\hline & & \multicolumn{3}{|c|}{$\begin{array}{l}\text { STANFORD UNIVERSITY } \\
\text { UNIVERSITY OF HAWAII }\end{array}$} \\
\hline & \multicolumn{3}{|c|}{ UNIVER SITY OF TOKYO } \\
\hline \multicolumn{2}{|c|}{$\begin{array}{l}\text { MONTANA STATE UNIVERSITY } \\
\text { UNIVERSITY OF NEVADA, RENO }\end{array}$} & \multicolumn{3}{|l|}{ UNIVER } \\
\hline \multicolumn{2}{|c|}{ NEW MEXICO STATE UNIVERSITY } & \\
\hline \multicolumn{2}{|c|}{ OREGON STATE UNIVERSITY } & \multicolumn{3}{|c|}{$\begin{array}{l}\text { WASHINGTON STATE UNIVERSITY } \\
\text { UNIVERSITY OF WASHINGTON }\end{array}$} \\
\hline
\end{tabular}

The Supporting Institutions listed above contribute to the cost of publication of this Journal, but they are not owners or publishers and have no responsibility for its content or policies.

\begin{abstract}
Mathematical papers intended for publication in the Pacific Journal of Mathematics should be in typed form or offset-reproduced (not dittoed), double spaced with large margins. Please do not use built up fractions in the text of the manuscript. However, you may use them in the displayed equations. Underline Greek letters in red, German in green, and script in blue. The first paragraph must be capable of being used separately as a synopsis of the entire paper. In particular it should contain no bibliographic references. Please propose a heading for the odd numbered pages of less than 35 characters. Manuscripts, in triplicate, may be sent to any one of the editors. Please classify according to the scheme of Math. Reviews, Index to Vol. 39. Supply name and address of author to whom proofs should be sent. All other communications should be addressed to the managing editor, or Elaine Barth, University of California, Los Angeles, California 90024.

There are page-charges associated with articles appearing in the Pacific Journal of Mathematics. These charges are expected to be paid by the author's University, Government Agency or Company. If the author or authors do not have access to such Institutional support these charges are waived. Single authors will receive 50 free reprints; joint authors will receive a total of 100 free reprints. Additional copies may be obtained at cost in multiples of 50 .
\end{abstract}

The Pacific Journal of Mathematics is issued monthly as of January 1966. Regular subscription rate: $\$ 190.00$ a year (5 Vols., 10 issues). Special rate: $\$ 66.00$ a year to individual members of supporting institutions.

Subscriptions, orders for numbers issued in the last three calendar years, and changes of address should be sent to Pacific Journal of Mathematics, P.O. Box 969, Carmel Valley, CA 93924, U.S.A. Old back numbers obtainable from Kraus Periodicals Co., Route 100, Millwood, NY 10546.

The Pacific Journal of Mathematics at P.O. Box 969, Carmel Valley, CA 93924 (ISSN 0030-8730) publishes 5 volumes per year. Application to mail at Second-class postage rates is pending at Carmel Valley, California, and additional mailing offices. Postmaster: Send address changes to Pacific Journal of Mathematics, P.O. Box 969, Carmel Valley, CA 93924.

\section{PUBLISHED BY PACIFIC JOURNAL OF MATHEMATICS, A NON-PROFIT CORPORATION}




\section{Pacific Journal of Mathematics}

\section{Vol. 116, No. 2 December, 1985}

Richard Arens, Reducing the order of the Lagrangean for a classical field in curved space-time ................................ 209

Shiferaw Berhanu, W. Wistar (William) Comfort and James Dolan Reid, Counting subgroups and topological group topologies $\ldots \ldots \ldots \ldots \ldots 217$

Michael James Cambern, Isomorphisms of spaces of norm-continuous

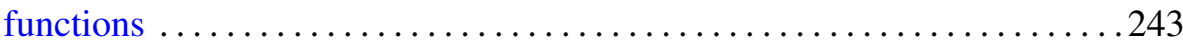

Gerald Arthur Edgar and Jun Feng Zhao, The ordering structure on

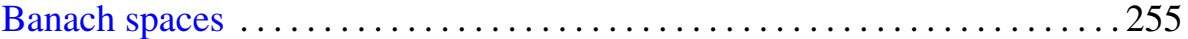

Irving Leonard Glicksberg, An analogue of Liapounoff's convexity theorem for Birnbaum-Orlicz spaces and the extreme points of their unit

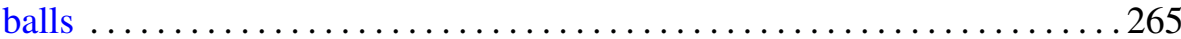

Daniel Heifetz, $p$-adic oscillatory integrals and wave front sets . ........285

José E. Pantoja, Liftings of supercuspidal representations of $\mathrm{Gl}_{2} \ldots \ldots \ldots 307$

Steven C. Pinault, An isoperimetric inequality for surfaces stationary with respect to an elliptic integrand and with at most three boundary

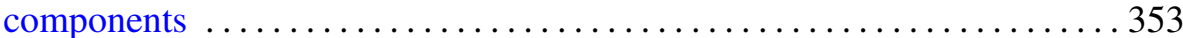

Georges Pinczon, Nonlinear multipliers and applications .............. 359 